\title{
QM/MM Investigation of the Spectroscopic Properties of the Fluorophore of Bacterial Luciferase
}

Germano Giuliani, ${ }^{\S}$ Federico Melaccio, ${ }^{\S}$ Samer Gozem, ${ }^{*}, \#$ Andrea Cappelli, ${ }^{\S}$ Massimo Olivucci.*,\$,

$\S$ Department of Biotechnology, Chemistry and Pharmacy, University of Siena, Via A. Moro 2, 53100 Siena (Italy).

\# Department of Chemistry, Georgia State University, Atlanta, GA 30302 (USA).

\$ Department of Chemistry, Bowling Green State University, Bowing Green, OH 43403 (USA).

\section{Supporting Information}




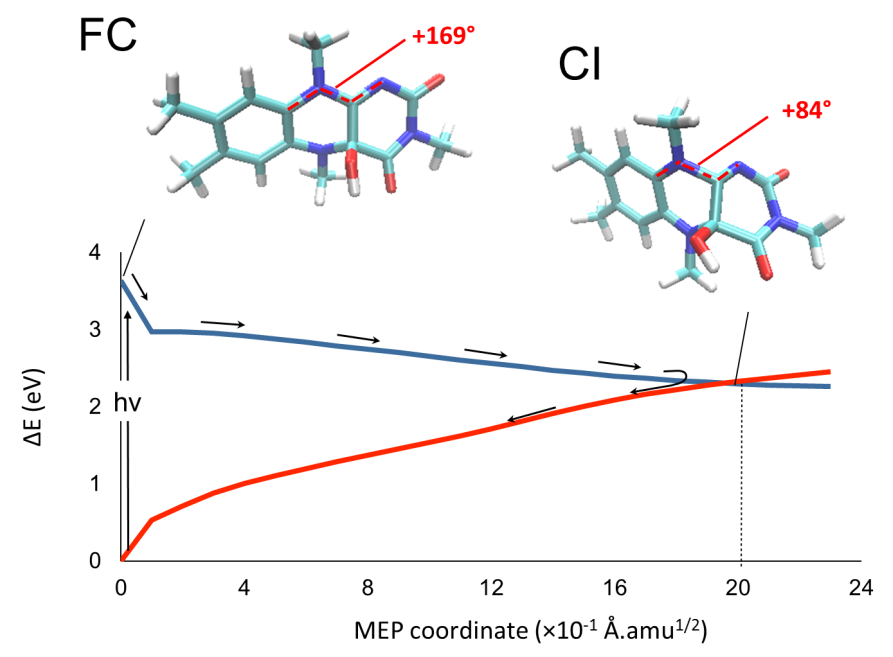

Figure S1. Computed $\mathrm{S}_{0}$ and $\mathrm{S}_{1}$ energy profiles of the N(3)- and N(5)-alkylated derivative of FMNHOH (2) in solution, connecting the Frank-Condon (FC) geometry to a Conical Intersection (CI). The arrows represent the internal conversion along the $\mathrm{S}_{1}$ pathway where the energy decrease is associated with an out-of-plane deformation of the pyrimidine ring with respect to the original flavin plane. The values of the geometric distortion which involves the $\mathrm{C}_{9 \mathrm{a}}-\mathrm{N}_{10}-\mathrm{C}_{10 \mathrm{a}}-\mathrm{N}_{1}$ dihedral angle of the molecule, are highlighted in red. Adapted from Gozem et al., Angew. Chem. Int. Ed. 2014, 53, 9870-9875.

\section{Preparing the protein model}

The crystallographic structure of Bacterial Luciferase (PDB code: $3 F G C)^{[1]}$ was downloaded from the Protein Data Bank. ${ }^{[2]}$ Charge parameters for the 4a-hydroxy flavin ( 2 in the main manuscript) were obtained using the restrained electrostatic potential (RESP) protocol derived from HF/6-31G* electron densities computed in Gaussian $03 .{ }^{[3]}$ Force constants for bonds, angles and torsions of $\mathbf{2}$ were taken from the GAFF parameter set. ${ }^{[4]}$ Preliminary energy minimization of the ligand in the gas phase was performed using the Molcas 7.8 program. ${ }^{[5]}$

The hydrogen atoms, counter ions and water solvent were added using GROMACS 4.6.3 ${ }^{[6]}$ employing the AMBER99SB force field for the protein ${ }^{[7]}$ and TIP3P force field for the crystallographic water molecules. The protonation states of ionized residue were assigned by estimating their $\mathrm{pKa}$ using PROPKA version 3.0. ${ }^{[8]}$ and assuming a neutral reference $\mathrm{pH}$. Estimated $\mathrm{pKa}$ 's are reported in Table $\mathrm{S} 1$, along with the reference $\mathrm{pKa}$ for each side chain in its standard protonation state (pKmodel). Residues with chosen nonstandard protonation states are highlighted in bold. Table S2 reports simulation parameters and the final 
choice of protonation states for specific ionizable residues which required a more detailed analysis. Following the PROPKA simulation, we used the following protocol:

- All Asp residues have been kept charged, since none had a pKa higher than 5.7;

- Glu43 and Glu328 were assigned a neutral protonation state because their pKa is close to 7 due to being buried inside the protein. Both had the extra hydrogen atom added to the OE2 carboxylate atom;

- Histidines were chosen to be neutral whenever their pKa was below 6. HID/HIE tautomer selection was based on visual inspection to create a reasonable local hydrogen bond network;

- Histidines with a pKa around 6 or higher were assigned the doubly protonated HIP states. His199 is an exception and was chosen to be neutral because of its short distance with positively charged Lys 202 .

Fourteen $\mathrm{Na}^{+}$anions were placed using the genion module of Gromacs to obtain a total zero charge for the model. The entire system (luciferase/FMNHOH complex) was solvated with a cubic TIP3P water box such that the edge of the water box is at least $6 \AA$ from the protein. In total, the system consists of 38985 atoms. Preliminary energy minimization was achieved with the protein (including the modelled mobile loop) and solvent with the ligand frozen during 1000-step minimization. Subsequently, a 5000-step energy minimization of part of the protein environment was performed, which includes all residues and water molecules within 8 Å of the fluorophore. 
Table S1

\begin{tabular}{|c|c|c|}
\hline Residue & pKa & pKmodel \\
\hline ASP 37 & 4.00 & 3.80 \\
\hline ASP 89 & 5.39 & 3.80 \\
\hline ASP 94 & 4.20 & 3.80 \\
\hline ASP 111 & 4.83 & 3.80 \\
\hline ASP 113 & 5.66 & 3.80 \\
\hline ASP 120 & 3.59 & 3.80 \\
\hline ASP 122 & 4.58 & 3.80 \\
\hline ASP 129 & 3.57 & 3.80 \\
\hline ASP 133 & 5.35 & 3.80 \\
\hline ASP 147 & 3.71 & 3.80 \\
\hline ASP 206 & 3.24 & 3.80 \\
\hline ASP 218 & 2.68 & 3.80 \\
\hline ASP 223 & 3.98 & 3.80 \\
\hline ASP 233 & 2.35 & 3.80 \\
\hline ASP 235 & 3.75 & 3.80 \\
\hline ASP 241 & 3.02 & 3.80 \\
\hline ASP 252 & 3.98 & 3.80 \\
\hline ASP 262 & 4.19 & 3.80 \\
\hline ASP 263 & 3.94 & 3.80 \\
\hline ASP 265 & 2.58 & 3.80 \\
\hline ASP 271 & 3.01 & 3.80 \\
\hline ASP 279 & 3.96 & 3.80 \\
\hline ASP 293 & 3.40 & 3.80 \\
\hline ASP 314 & 3.44 & 3.80 \\
\hline ASP 316 & 3.45 & 3.80 \\
\hline ASP 321 & 4.62 & 3.80 \\
\hline ASP 346 & 3.16 & 3.80 \\
\hline GLU 14 & 4.11 & 4.50 \\
\hline GLU 19 & 3.68 & 4.50 \\
\hline GLU 32 & 4.16 & 4.50 \\
\hline GLU 43 & $\mathbf{8 . 7 5}$ & 4.50 \\
\hline GLU 48 & 4.02 & 4.50 \\
\hline GLU 67 & 4.58 & 4.50 \\
\hline GLU 88 & 4.92 & 4.50 \\
\hline GLU 137 & 4.92 & 4.50 \\
\hline GLU 141 & 4.69 & 4.50 \\
\hline GLU 149 & 4.32 & 4.50 \\
\hline GLU 175 & 5.68 & 4.50 \\
\hline GLU 181 & 4.91 & 4.50 \\
\hline GLU 185 & 4.36 & 4.50 \\
\hline GLU 200 & 4.11 & 4.50 \\
\hline GLU 210 & 4.77 & 4.50 \\
\hline
\end{tabular}

\begin{tabular}{|c|c|c|}
\hline GLU 214 & 4.53 & 4.50 \\
\hline GLU 297 & 4.77 & 4.50 \\
\hline GLU 305 & 4.74 & 4.50 \\
\hline GLU 306 & 3.33 & 4.50 \\
\hline GLU 328 & 6.67 & 4.50 \\
\hline GLU 333 & 4.82 & 4.50 \\
\hline GLU 334 & 4.66 & 4.50 \\
\hline GLU 335 & 3.97 & 4.50 \\
\hline GLU 353 & 3.67 & 4.50 \\
\hline C- 355 & 3.28 & 3.20 \\
\hline HIS 44 & 2.23 & 6.50 \\
\hline HIS 45 & 3.96 & 6.50 \\
\hline HIS 61 & 6.17 & 6.50 \\
\hline HIS 82 & 5.07 & 6.50 \\
\hline HIS 150 & 6.00 & 6.50 \\
\hline HIS 199 & 6.37 & 6.50 \\
\hline HIS 215 & 7.01 & 6.50 \\
\hline HIS 224 & 3.36 & 6.50 \\
\hline HIS 234 & 6.48 & 6.50 \\
\hline HIS 249 & 6.32 & 6.50 \\
\hline CYS 34 & 10.17 & 9.00 \\
\hline CYS 106 & 13.73 & 9.00 \\
\hline CYS 130 & 11.27 & 9.00 \\
\hline CYS 225 & 19.25 & 9.00 \\
\hline CYS 243 & 12.08 & 9.00 \\
\hline CYS 307 & 12.62 & 9.00 \\
\hline CYS 324 & 9.29 & 9.00 \\
\hline CYS 325 & 12.93 & 9.00 \\
\hline TYR 10 & 11.77 & 10.00 \\
\hline TYR 56 & 15.37 & 10.00 \\
\hline TYR 110 & 14.16 & 10.00 \\
\hline TYR 132 & 12.35 & 10.00 \\
\hline TYR 143 & 9.92 & 10.00 \\
\hline TYR 163 & 10.16 & 10.00 \\
\hline TYR 171 & 15.03 & 10.00 \\
\hline TYR 208 & 13.06 & 10.00 \\
\hline TYR 217 & 10.52 & 10.00 \\
\hline TYR 228 & 16.66 & 10.00 \\
\hline TYR 251 & 10.22 & 10.00 \\
\hline TYR 254 & 10.75 & 10.00 \\
\hline TYR 270 & 10.14 & 10.00 \\
\hline TYR 294 & 10.30 & 10.00 \\
\hline TYR 296 & 10.82 & 10.00 \\
\hline
\end{tabular}

\begin{tabular}{|c|c|c|}
\hline TYR 350 & 10.31 & 10.00 \\
\hline LYS 2 & 11.05 & 10.50 \\
\hline LYS 22 & 10.60 & 10.50 \\
\hline LYS 29 & 10.31 & 10.50 \\
\hline LYS 98 & 10.52 & 10.50 \\
\hline LYS 112 & 9.25 & 10.50 \\
\hline LYS 136 & 9.42 & 10.50 \\
\hline LYS 152 & 10.59 & 10.50 \\
\hline LYS 155 & 10.40 & 10.50 \\
\hline LYS 201 & 9.21 & 10.50 \\
\hline LYS 202 & 11.25 & 10.50 \\
\hline LYS 221 & 10.65 & 10.50 \\
\hline LYS 240 & 10.59 & 10.50 \\
\hline LYS 259 & 10.56 & 10.50 \\
\hline LYS 268 & 9.77 & 10.50 \\
\hline LYS 274 & 10.59 & 10.50 \\
\hline LYS 283 & 10.48 & 10.50 \\
\hline LYS 341 & 10.56 & 10.50 \\
\hline LYS 352 & 10.45 & 10.50 \\
\hline LYS 354 & 10.33 & 10.50 \\
\hline ARG 23 & 11.80 & 12.50 \\
\hline ARG 85 & 12.53 & 12.50 \\
\hline ARG 100 & 11.51 & 12.50 \\
\hline ARG 102 & 11.55 & 12.50 \\
\hline ARG 107 & 10.56 & 12.50 \\
\hline ARG 115 & 12.26 & 12.50 \\
\hline ARG 125 & 11.23 & 12.50 \\
\hline ARG 186 & 12.53 & 12.50 \\
\hline ARG 238 & 14.28 & 12.50 \\
\hline ARG 244 & 13.49 & 12.50 \\
\hline ARG 278 & 12.47 & 12.50 \\
\hline ARG 290 & 12.44 & 12.50 \\
\hline ARG 291 & 12.74 & 12.50 \\
\hline N+ 1 & 8.85 & 8.00 \\
\hline
\end{tabular}


Table S2

\begin{tabular}{|c|c|c|c|c|}
\hline Residue & $p K a$ & Buried (\%) & Hydrogen bond & Chosen Protonation \\
\hline HIS44 & 2.2 & $81 \%$ & - & HID \\
\hline HIS45 & 4.0 & $70 \%$ & GLH43 & HID \\
\hline HIS61 & 6.2 & $2 \%$ & - & HIE \\
\hline HIS82 & 5.1 & $53 \%$ & - & HIE \\
\hline HIS150 & 6.0 & $29 \%$ & - & HID \\
\hline HIS199 & 6.4 & $0 \%$ & - & HID \\
\hline HIS215 & 7.0 & $0 \%$ & - & HIP \\
\hline HIS224 & 3.4 & $99 \%$ & - & HID \\
\hline HIS234 & 6.5 & $0 \%$ & - & HIP \\
\hline HIS249 & 6.3 & $8 \%$ & - & HIP \\
\hline HIS285 & 6.1 & $0 \%$ & - & HIP \\
\hline GLU43 & 8.8 & $100 \%$ & HID45 & GLH:OE2 \\
\hline GLU328 & 6.7 & $100 \%$ & - & GLH:OE2 \\
\hline ARG290 & 12.4 & $0 \%$ & GLU200 & ARG:NH1 \\
\hline
\end{tabular}




\section{Replica-exchange molecular dynamics (REMD) simulations}

REMD simulations were performed using the GROMACS 4.6 .3 program. ${ }^{[6]}$ The loop and all residues and water molecules having at least an atom within $6 \AA$ of the loop residues were allowed to move, while the rest of the system was kept fixed. This is because the missing loop is a highly mobile domain and had to be better sampled to ensure a comparable accuracy with the rest of the MM model derived from crystallography. ${ }^{[9]}$ All classical and REMD trajectories were carried out in the NVT ensemble with a Berendsen thermostat. ${ }^{[10]}$ Preliminary standard MD simulations (Figure S2 and S3, split into two figures for clarity) were performed separately with temperatures distributed between 280 and $347 \mathrm{~K}$. The simulations were conducted for $1.5 \mathrm{~ns}$ with the annealing-temp option for each temperature. The REMD simulation (Figure S4) was set up with replica exchange between the different temperatures every 2 picosecond, during $10 \mathrm{~ns}$ of dynamics at reference temperature of $298 \mathrm{~K}$. The temperature of the system was controlled using Langevin dynamics with collision frequency $(\gamma)$ of 1 ps.

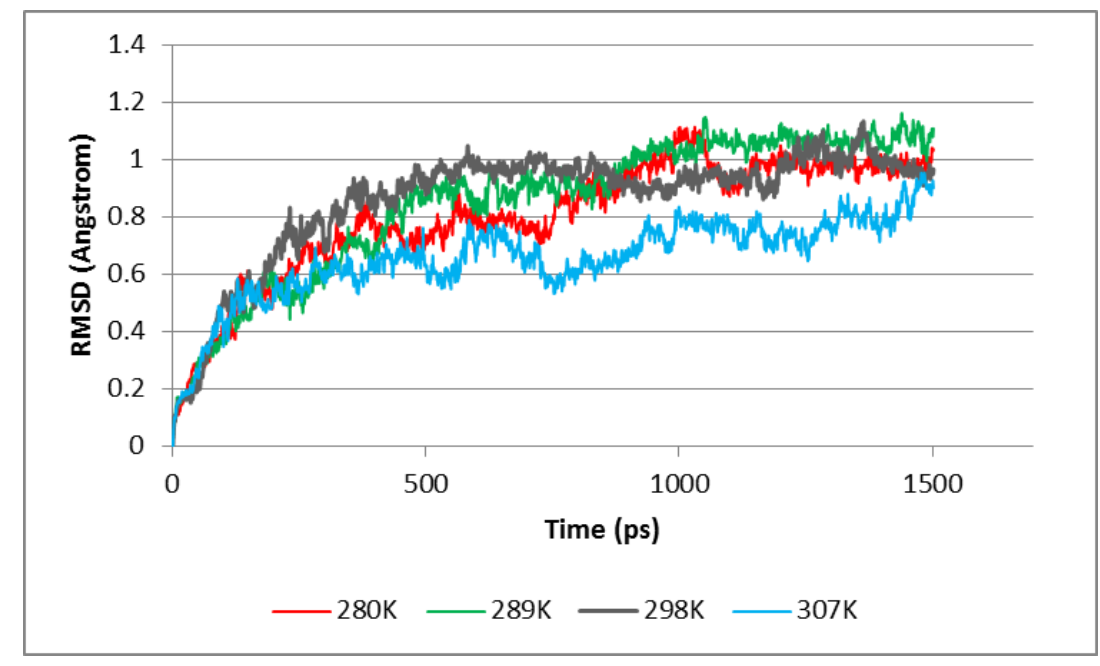

Figure S2. Molecular Dynamic trajectories obtained at different equilibration temperatures. 


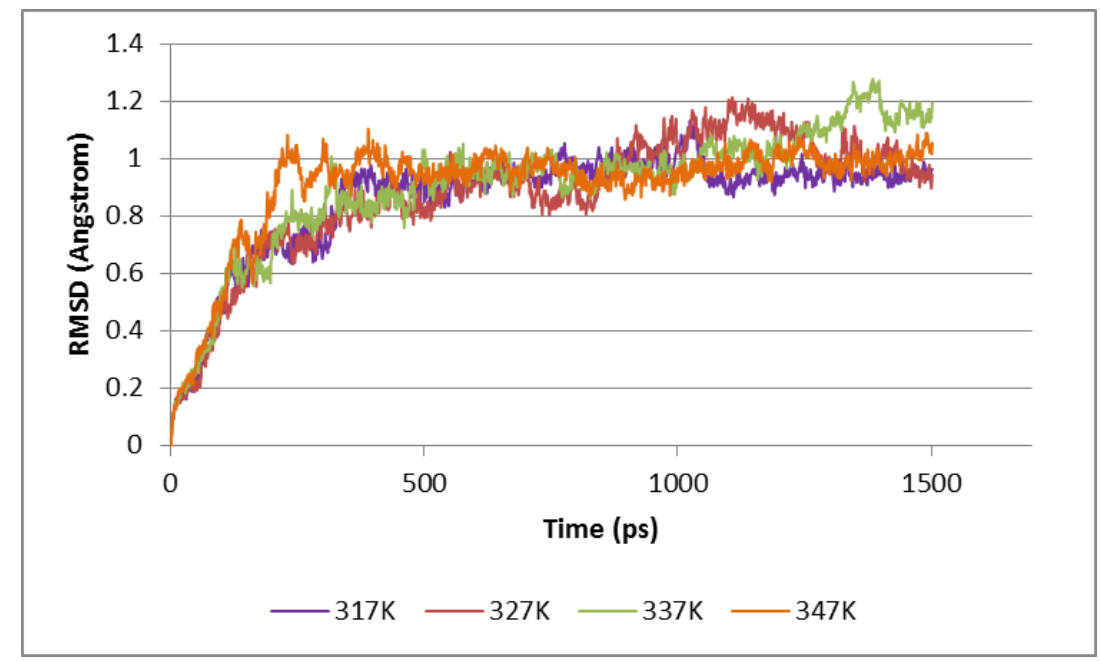

Figure S3. Molecular Dynamic trajectories obtained at different equilibration temperatures.

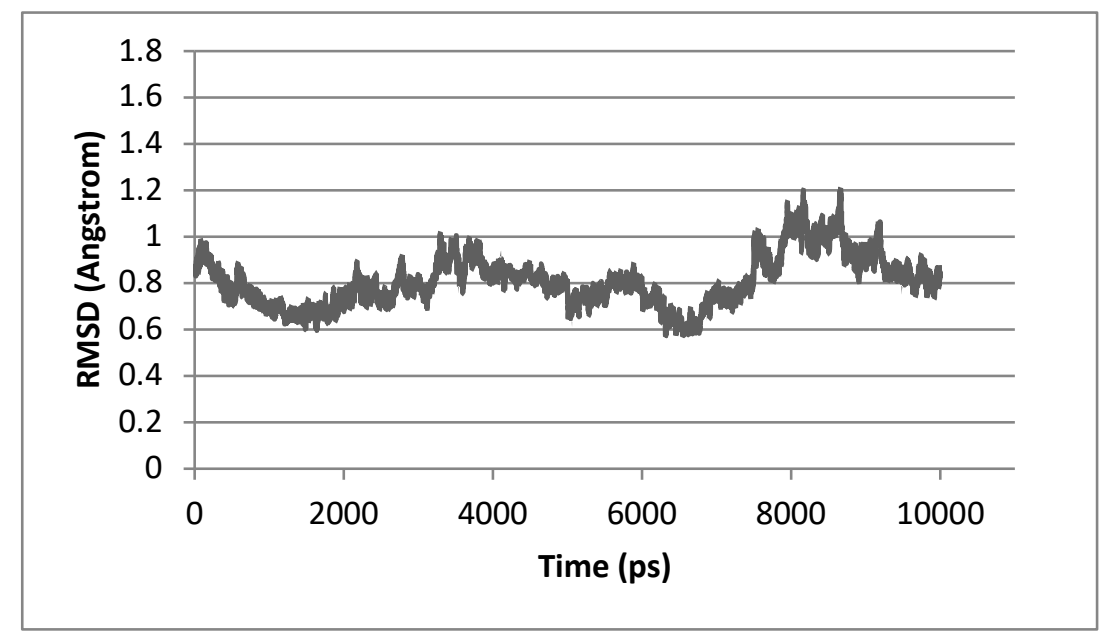

Figure S4. REMD trajectory obtained at reference temperature of $298 \mathrm{~K}$. RMSD $\leq 0.6 \AA$.

\section{Cluster analysis of REMD simulations}

REMD simulations make it possible to substantially increase the conformational space sampled by molecular dynamics. However, the interpretation of results requires statistical analysis. Our aim was to find the most populated loop conformation under the assumption that it is the preferred one in physiological conditions. A well-established tool is cluster analysis, which has been routinely used for this purpose. We used the singlelinkage algorithm ${ }^{[11]}$ as implemented in the GROMACS 4.6.3 $g_{-}$cluster routine, with a $0.1 \mathrm{~nm}$ RMSD cutoff. The analysis was performed on the REMD de-multiplexed trajectory at $298 \mathrm{~K}$ constant-temperature (i.e. our 
reference trajectory, Figure S4). 96 clusters were obtained, with only 5 clusters including more than 500 MD trajectory snapshots. Figure S5 show the number of structures for each obtained cluster.

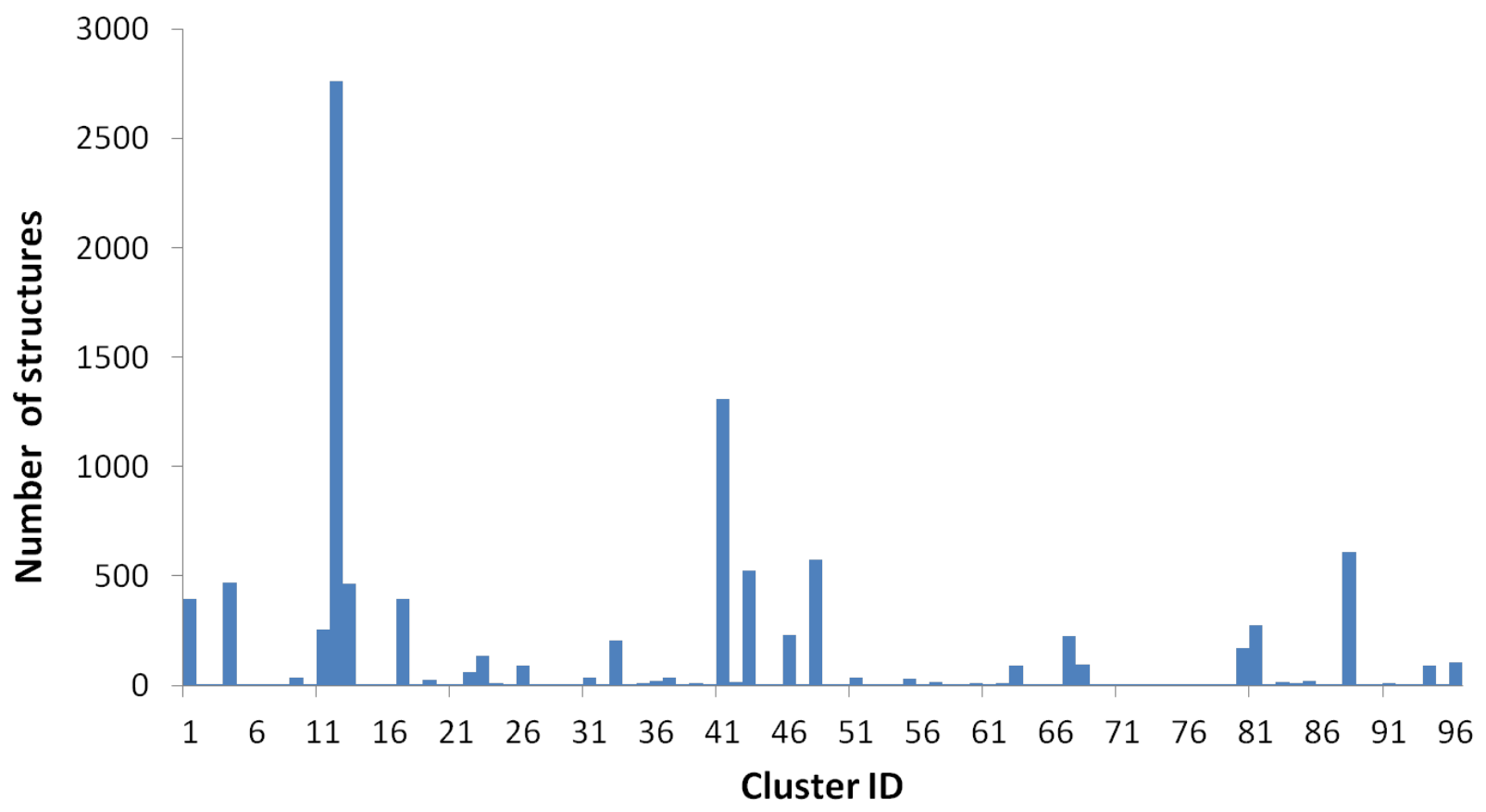

Figure S5. Number of structures for each cluster resulting from our analysis.

Cluster \#12 was the most populated one with 2765 snapshots, while the second most populated is cluster \#41 with less than half of cluster \#12 frames. The reference structure used for subsequent QM/MM calculations has been chosen as the snapshot in cluster \#12 with the lowest RMSD from the average structure produced by the $g_{-}$cluster routine. This is the MD snapshot that would resemble the cluster "average structure" more closely, while being a realistic structure, instead of the actual average which might include chemical artifacts.

To assess the effect of the chosen RMSD cutoff on the resulting model, we have performed other cluster analyses to plot the number of obtained clusters as a function of the RMSD cutoff. The optimal value would be a compromise between a too low one which produces many clusters with a population of 1 or fewmembers clusters or a too large value which groups most structures in the same cluster, both of which are meaningless. As seen in Figure S6, the $0.1 \mathrm{~nm}$ cutoff marks the beginning of the flat section of the curve, which is the region where cluster analysis would generate non-trivial clusters. The most populated clusters 
should remain the same throughout that region, regardless of the chosen cutoff. While cutoff values of 0.115 and $0.120 \mathrm{~nm}$ lead to a very large "best" clusters comprising $77 \%$ and $94 \%$ of the trajectory respectively, 0.105 and $0.11 \mathrm{~nm}$ would be suitable choices too, given the even lower number of obtained clusters. Therefore, we performed the extraction of the snapshot closest to the cluster average structure for the most populated clusters obtained with 0.105 and $0.11 \mathrm{~nm}$ cutoff values. Both these structures belong to the most populated cluster from our original cluster analysis (i.e. with $0.1 \mathrm{~nm}$ cutoff). Furthermore, our reference structure is present in the most populated clusters in both cases, which confirms that the favored loop conformation is independent of the RMSD cutoff.

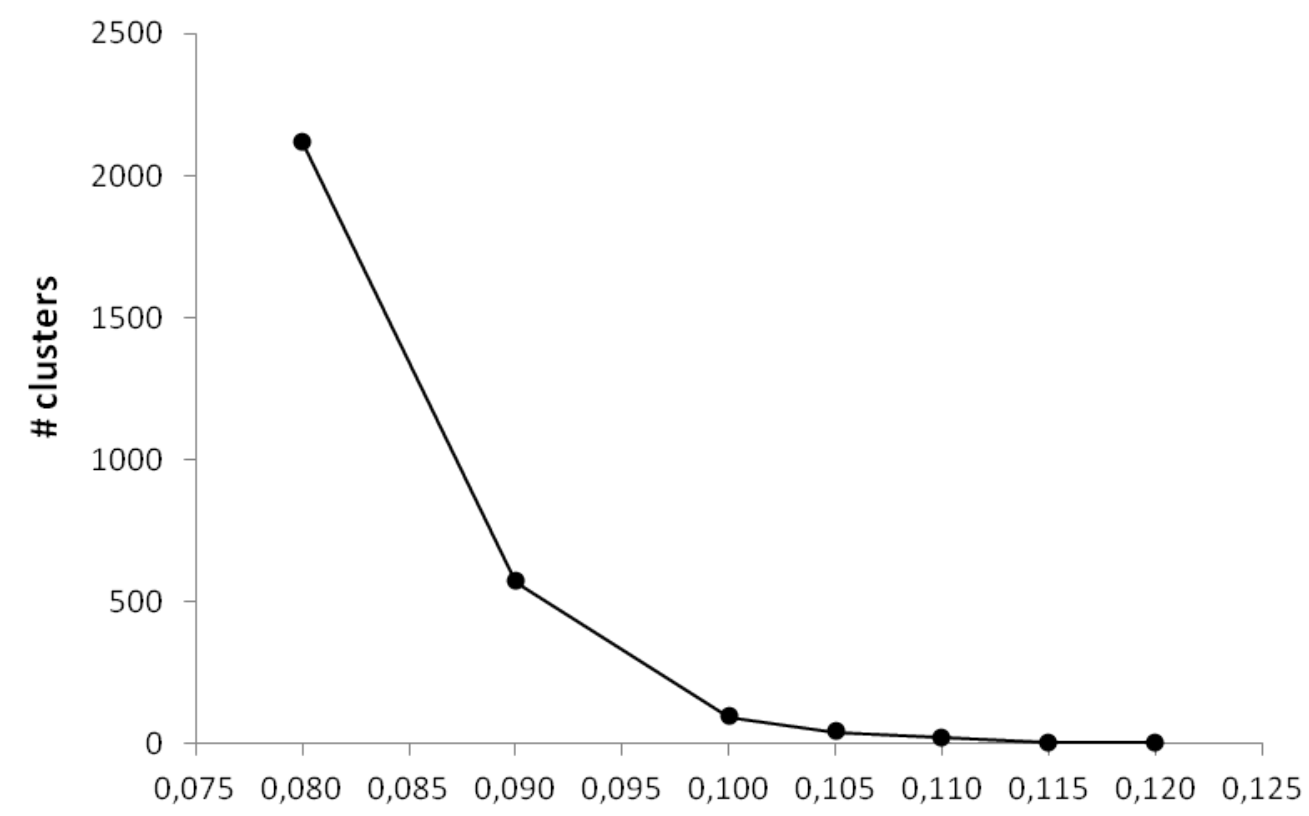

\section{RMSD cutoff $(\mathrm{nm})$}

Figure S6. Number of obtained clusters as a function of RMSD cutoff values. 


\section{QM/MM Calculations}

Optimizations and MEP calculations for FMNHOH (2), embedded in the protein environment, were performed at the 3-root state-averaged CASSCF level of theory ${ }^{[12]}$ with a $6-31 \mathrm{G}^{*}$ basis set. The CASSCF wave function comprised an active space of 14 electrons in 11 orbitals (see Figure S7 for a full description of the active space). These orbitals include $\pi$ and $\pi^{*}$ orbitals involved in the $\pi$-conjugated system of FMNHOH. The full active space (i.e. including all $\pi$ and $\pi *$ orbitals) would comprise 18 electrons and 15 orbitals, but here we use a slightly reduced active space of 14 electrons and 11 orbitals such that 4 orbitals and electrons have been excluded from the active space due to the high computational cost of using the full active space. We do, however, check the effect of using the full 18,15 active space on the spectroscopic properties of FMNHOH (see manuscript). The excluded electrons and orbitals belong to lower energy $\pi$ and higher energy $\pi^{*}$ orbitals of the benzene ring. Non-bonding orbitals are excluded from the active space since $n, \pi^{*}$ states are assumed to be high in energy, based on earlier TD-DFT studies, ${ }^{[13]}$ and are also not relevant to the spectroscopic properties of the system (they are dark states).

Spectroscopic properties were computed using several different flavors of CASPT2 and basis sets. We test the effect of the IPEA shift (0 vs. 0.25$)$, the basis set (6-31G*, ANO-L-VDZP, and ANO-L-VTZP), active space $(18,15$ vs. 14,11$)$ and state-averaging (SS vs MS CASPT2).

Two models were generated; one with a cysteine (Cys106) included in the QM calculation, and one where it is treated at the MM level. Even with Cys106 in the QM region, however, no cysteine orbitals were included in the active space (Fig. S7).

The MEPs were computed in the form of an intrinsic reaction coordinate as implemented in Molcas 7.8 , with a step constraint of 0.03 Bohr.amu ${ }^{1 / 2}$ for the first eight steps, followed by 0.05 Bohr.amu $^{1 / 2}$ for the remainder of the MEP. Single point CASPT2 calculations ${ }^{[14]}$ were then performed for stationary points and for each point along the MEP path. Our CASPT2 calculations do not include an IPEA shift ${ }^{[15]}$ and employ the same basis set and active space as in the CASSCF calculations $\left(14,11\right.$ and $\left.6-31 G^{*}\right)$. All calculations were performed with Molcas v. 7.8. ${ }^{[5]}$ 


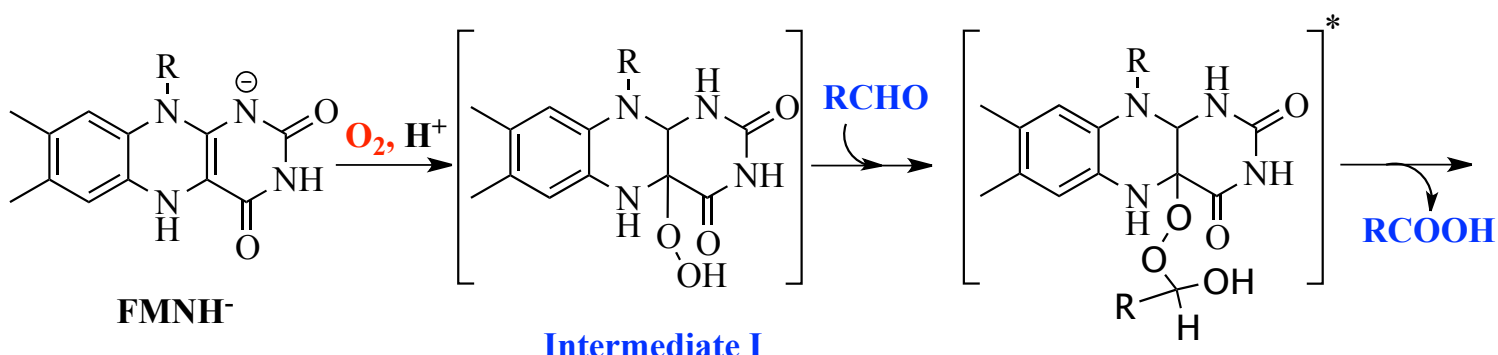

Intermediate I

Intermediate II

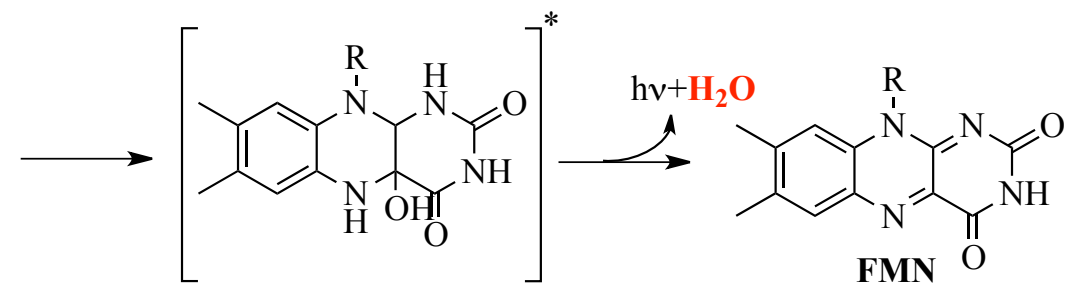

Intermediate III

Figure S7: Proposed mechanism for bioluminescence in bacterial luciferase. Adapted from ref. [16] The lowest-energy absorption band of 4a-hydroxy flavins are taken as the signature for the participation of a 4ahydroxy flavin intermediate (e.g. Intermediate III) to the catalytic cycle of various flavoprotein monooxygenases (such as styrene monooxygenase ${ }^{[17]}$ and p-hydroxybenzoate hydroxylase ${ }^{[18]}$ )
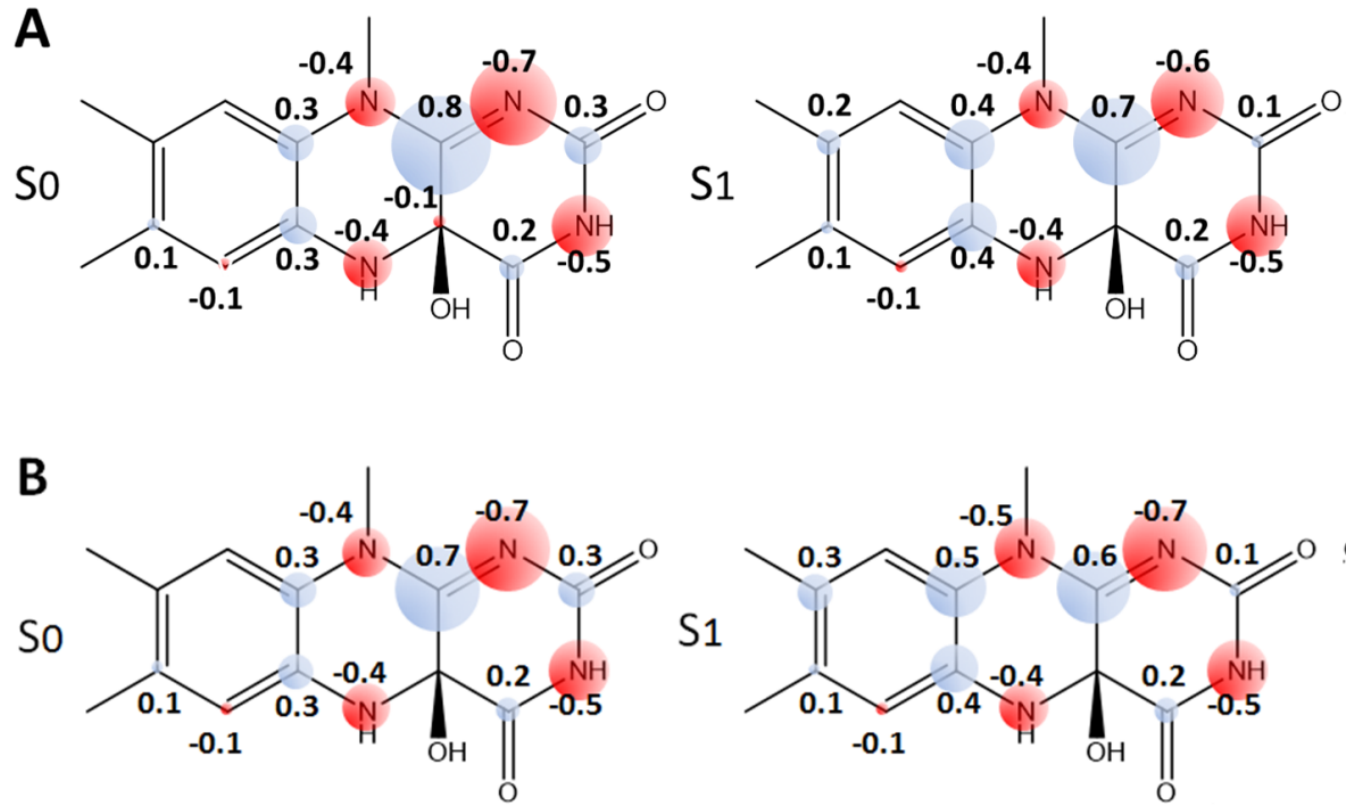

Figure S8: CASPT2 Mulliken charge distributions for FMNHOH at select geometries. Charges are shown for the ground state $\left(\mathrm{S}_{0}\right.$, left), and the first singlet excited state $\left(\mathrm{S}_{1}\right.$, right) for: A. The Frank-Condon geometry. B. The energy minimum (EM) geometry. The charges of all hydrogen atoms and other substituents were summed onto the atoms involved in the backbone ring structure of FMNHOH. 

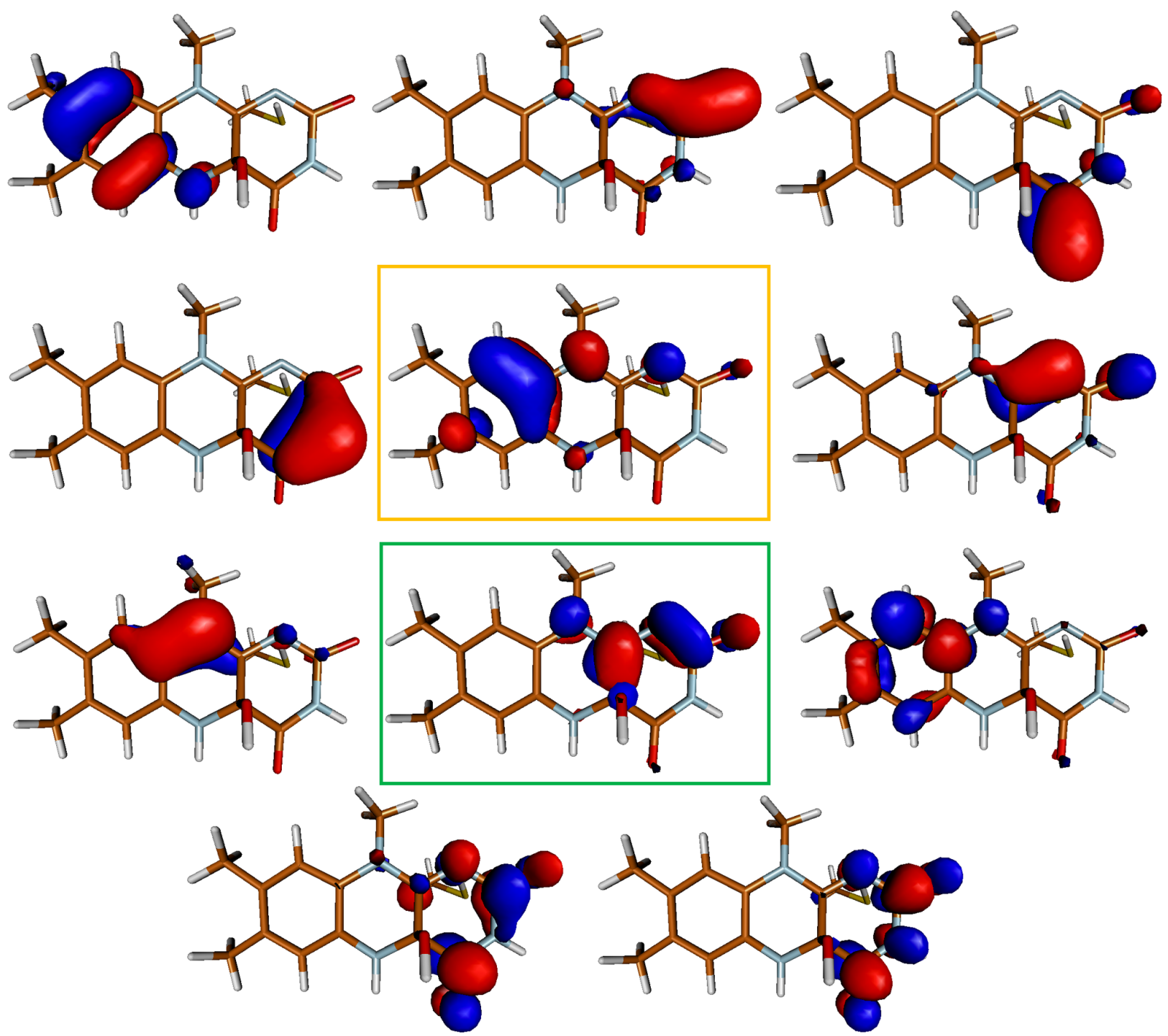

Figure S9: The active space orbitals used in CASSCF and CASPT2 calculations with the 6-31G* basis set. Shown here are the active space orbitals for the calculation with Cys106 in the QM region. The same orbitals were used in the calculation with Cys 106 treated with MM. The 18,15 calculations include the full set of valence $\pi$ and $\pi^{*}$, i.e., including two $\pi$ and two $\pi^{*}$ orbitals on benzene missing above. The HOMO (orange frame) and LUMO (green frame) orbitals are highlighted. 
Coordinates and absolute CASSCF, 6-31G* QM/MM energies of stationary points:

Ground state optimized geometry (GS)

35

N 35.0414667440 .0283599134 .23404388

C 34.6003929640 .0165720632 .95813985

O 33.4613197840 .2818299032 .65701521

N 35.4641449839 .6242475431 .92657785

C 36.7987885639 .4501424532 .06361589

O 37.5308168739 .0790232631 .17914975

C 37.3732803639 .8672053133 .40507003

N 38.3682150738 .9391856133 .77664625

C 38.9566060939 .1678134335 .03067914

C 40.2451758238 .7951940635 .31448025

C 40.7917406138 .9515161236 .59100268

C 42.1867400238 .4393108036 .87381508

C 40.0183484539 .5667991037 .57449053

C 40.4951262739 .7239231239 .00080337

C 38.7302363939 .9943897137 .26685293

C 38.1651610839 .7668430636 .02038938

N 36.7949452140 .0028706635 .73061068

C 36.3229098539 .9409389434 .49712057

C 35.8813826640 .3145438336 .83917113

H 35.0787883239 .5393919731 .01048163

H 40.8254196138 .3344537334 .53144316

H 42.1899898637 .7147395137 .68144759

H 42.6064995037 .9615285835 .99649902

H 42.8658861139 .2369095137 .15810035

H 40.0085204040 .5653858839 .47937486

H 40.2439518538 .8356353439 .57717561

H 41.5686029339 .8673321639 .05761895

H 38.1663765040 .4687819838 .04582401

H 36.2122403941 .2355246737 .29580134

H 34.8894623340 .4433453236 .45107810

H 39.0276180738 .7091070133 .05738640

O 37.7623775941 .2045585233 .26477724

H 38.4694401741 .2758006032 .63396544

C 35.8789554939 .2209740237 .87485282

H 35.8796011139 .5118635937 .59936149 
Excited state minimum geometry (EM)

35

$-946.210866308881$

N 35.0010271639 .9967609934 .22691256

C 34.5634454839 .9812998032 .98723757

O 33.4221718440 .2680544132 .61313145

N 35.4643218039 .5679958431 .95006172

C 36.7840460139 .4015006432 .08849009

O 37.5498013039 .0049897031 .22895376

C 37.3156621839 .8785976133 .42552272

N 38.5169248439 .1627343033 .74946906

C 38.9641776939 .1898075835 .00495771

C 40.2719882738 .7397082535 .29989879

C 40.8082680938 .8150054736 .55768862

C 42.1914497238 .2747550336 .83353825

C 40.0331093039 .4176838637 .57247500

C 40.4914823739 .5377846938 .99234834

C 38.7090749739 .8828416637 .28006506

C 38.1096843539 .6908508036 .05076865

N 36.7766052839 .9551427535 .79227507

C 36.2786976239 .6849796834 .49831954

C 35.8772093440 .2809221236 .88264175

H 35.0705696439 .4105794431 .04793483

H 40.8412605738 .3343179734 .48086214

H 42.1662237237 .5028065637 .59512887

H 42.6259870737 .8499563535 .93751214

H 42.8628827439 .0519017737 .18071558

H 40.1160508740 .4594468039 .42599874

H 40.0737209538 .7094268339 .57268559

H 41.5711278639 .5020935839 .07276042

H 38.1790648940 .3557080038 .08352114

H 36.1864188141 .2050956437 .35835526

H 34.8909963840 .4189644936 .47727757

H 39.1399455538 .8746501933 .01975805

O 37.5436874441 .2545139433 .33926660

H 38.1280839441 .4471271632 .61548464

C 35.8351031339 .1926018937 .92980650

H 35.8463033839 .4820950737 .65126068 


\section{References}

1. Z.T. Campbell, A. Weichsel, W.R. Montfort, T.O. Baldwin, Biochemistry 2009, 48, 6085-6094.

2. http://www.rcsb.org/.

3. P. Cieplak, W.D. Cornell, C. Bayly, P.A. Kollman, J. Comput. Chem. 1995, 16, 1357-1377.

4. J.M. Wang, R.M. Wolf, J.W. Caldwell, P.A. Kollman, D.A. Case J. Comput. Chem. 2004, 25, 11571174.

5. F. Aquilante, L. De Vico, N. Ferré, G. Ghigo, P. A. Malmqvist, P. Neogrády, T. B. Pedersen, M. Pitonák, M. Reiher, B. O. Roos, L. Serrano-Andrés, M. Urban, V. Veryazov, and R. Lindh, J. Comput. Chem. 2010, 31, 224-47.

6. a) H.J.C. Berendsen, D. Van der Spoel, R. Van Drunen, Comp. Phys. Comm. 1995, 91, 43-56. b) E. Lindhal, B. Hess, D. Van der Spoel, J. Mol. Model. 2001, 7, 306-317.

7. V. Hornak, R. Abel, A. Okur, B. Strockbine, C. Simmerling, Proteins, 2006, 65, 712-725.

8. PROPKA 3 Mats H.M. Olsson, Chresten R. Sondergard, Michal Rostkowski, and Jan H. Jensen, J. Chem. Theory Comput. 2011, 7, 525-537.

9. A. Onufriev, D. Bashford, D.A. Case, Proteins 2004, 55, 383-394. J. Chen, C.L. Brooks, J. Khandogin, Curr. Opin. Struct. Biol. 2008, 18, 140-148.

10. G. Bussi, D. Donadio and M. Parrinello, J. Chem. Phys. 2007, 126, 014101.

11. A. E. Torda, W. F. van Gusteren, J. Comput. Chem.1994, 15, 1331-1340.

12. B. O. Roos, in Advances in Chemical Physics: Ab Initio Methods in Quantum Chemistry Part 2, Volume 69 (Ed.: K.P. Lawley), Wiley Online Library, 1987, p 399-445.

13. D. Zhou, E. Mirzakulova, R. Khatmullin, I. Schapiro, M. Olivucci, K. D. Glusac, J. Phys. Chem. B 2011, 115, 7136-43.

14. K. Andersson, P. A. Malmqvist, B. O. Roos, A. J. Sadlej, K. Wolinski, J. Phys. Chem. 1990, 94, 5483-5488.

15. G. Ghigo, B. O. Roos, P. K. Malmqvist, Chem. Phys. Lett. 2004, 396, 142-149.

16. B. Lei, Q. Ding, S. C. Tu, Biochemistry 2004, 43, 15975-82.

17. a) A. Kantz, G. T. Gassner, Biochemistry 2011, 50, 523-32. b) D. Tischler, M. Schlömann, W. J. van Berkel, G. T. Gassner, FEBS letters 2013, 587, 3848-3852.

18. B. Entsch, D. P. Ballou, V. Massey, J. Biol. Chem. 1976, 251, 2550-63. 